\title{
Comparison of different analytic heliospheric magnetic field configurations and their significance for the particle injection at the termination shock
}

\author{
K. Scherer ${ }^{1}$, H. Fichtner ${ }^{1}$, F. Effenberger ${ }^{1}$, R. A. Burger ${ }^{2}$, and T. Wiengarten ${ }^{1}$ \\ 1 Institut für Theoretische Physik IV, Ruhr Universität Bochum, 44780 Bochum, Germany \\ e-mail: kls@tp4.rub.de \\ 2 Unit for Space Physics, North-West University, 2520 Potchefstroom, South Africa
}

Received 11 November 2009 / Accepted 19 May 2010

ABSTRACT

\begin{abstract}
Context. Heliospheric magnetic field configurations are relevant on a large scale for the transport of cosmic rays, especially for injecting possibly pre-accelerated particles into the Fermi-I acceleration process of cosmic rays.

Aims. We compare four analytically described fields by calculating the scalar product between their vectors and the corresponding vectors of the Parker spiral field. Because the injection efficiency at the termination shock is highly sensitive to the local inclination of the upstream magnetic fields with respect to the shock normal, we discuss the probability that pick-up ions are injected into the Fermi-I process.

Methods. We extend the previous work comparing different analytically described heliospheric magnetic fields: the Parker and hybrid Fisk field and modifications of both. Because the Fisk-like configurations are only present during periods of low to moderate solar activity, we restrict ourselves to this case by including high-speed streams over the ecliptic and low-speed flow in the latter. In addition, we extend the analysis from an analytic approximation to a numerically computed termination shock surface.

Results. We find that no strong differences in the injection efficiency can be expected for the four field configurations despite significant structural differences inside the termination.

Conclusions. Consequently, the injection efficiency is largely insensitive to the large-scale heliospheric magnetic field configuration.
\end{abstract}

Key words. magnetic fields - solar wind - acceleration of particles

\section{Introduction}

The heliospheric magnetic field (HMF) is important for the transport of cosmic rays and especially for the injection of pickup ions (PUIs) into the Fermi-I acceleration process at the termination shock to become anomalous cosmic rays. There are several approaches to describing this field analytically, starting with Parker (1958), who assumed a freezing-in of the magnetic field into the solar wind, which leads to an Archimedian spiral, except at the poles where the field is purely radial. To avoid such a radial field line over the poles, Jokipii \& Kota (1989) added a small correction term to the latitudinal component. Similarly, Smith \& Bieber (1991) added an additional azimuthal contribution (see also Burger 2005) to describe the under- and overwinding of the HMF close to the ecliptic. Later, Fisk (1996) modeled the HMF and included the footpoint motion of field lines on the Sun's differentially rotating surface. The assumption of a constant solar wind speed in this field, was abandoned by Schwadron (2002), see also Schwadron et al. (2008). For a deeper discussion of these HMFs, see Burger \& Hitge (2004), Burger (2005), and Burger et al. (2008).

The existing observational data (see e.g. Burlaga et al. 2002; Murphy et al. 2002; Mursula 2007; Roberts et al. 2007; Mordvinov 2008; Ebert et al. 2009) and corresponding modeling (e.g. Burger \& Hitge 2004; Giacalone \& Jokipii 2004; Burger 2005; Schwadron \& McComas 2005; Giacalone et al. 2006; Ferreira et al. 2007; Burger et al. 2008) that can be used to analyze the asymmetry of the HMF have not yet convincingly settled the debate over which of the fields is closest to reality. For a more detailed discussion of the difficulties to distinguish between different fields on the basis of in situ measurements of magnetic fields, see the recent paper by Hitge \& Burger (2010).

A direct and meaningful comparison of three-dimensional vector fields is not a straightforward task. We discuss the angle between the Parker field and the above-mentioned fields with respect to heliocentric distance $r$, latitude $\vartheta$, and longitude $\varphi$. Furthermore, we use the analytic representation of the different magnetic tilt angles against $r, \varphi$, and $\vartheta$. We compare the global structure of the four analytic HMF models mentioned above, especially at the termination shock, and discuss the consequences for particle injection. Because this injection of PUIs at the termination shock into the Fermi-I acceleration process strongly depends on the magnetic tilt angle between the magnetic field and the shock surface normal, we analyze the tilt angle for the magnetic field configurations. This is first done in the same way as in Fahr et al. (2008) and Scherer \& Fahr (2009), by describing the 3D-shape of the termination shock by the surface of a triaxial ellipsoid fitted to a shock, which was numerically computed by Borrmann \& Fichtner (2005). Second, we extend this analysis by using the actual shock surface rather than its analytical approximation.

In Sect. 2 we summarize the analytic descriptions of the different HMFs, and in Sect. 3 we discuss the differences of their direction angles with respect to the Parker field. In Sect. 4 we briefly introduce a triaxial ellipsoid as an analytic approximation of the termination shock (Scherer \& Fahr 2009) and a numerical 
representation of it. Finally in Sect. 5 we briefly discuss consequences for particle injection and propagation, as well as for the fluxes of energetic neutral atoms (ENAs) produced by PUIs (see Scherer \& Fahr 2003; Sternal et al. 2008a; Heerikhuisen et al. 2008).

\section{The different heliospheric magnetic fields}

Modeling the time-dependent heliosphere in three dimensions requires adequate modeling of solar-cycle related high-speed streams in the heliosphere. This modeling includes the interaction of interstellar protons and neutral gas with the supersonic solar wind protons (e.g. Borrmann \& Fichtner 2005) and involved magnetic fields (Opher et al. 2006; Ratkiewicz 2006; Pogorelov et al. 2009), and also includes both cosmic rays (Fahr et al. 2000; Ferreira et al. 2007) and time-dependent dynamical effects of the solar activity cycle (Scherer \& Ferreira 2005; Izmodenov et al. 2008). Here we do not follow up on these topics, but refer the reader to Müller et al. (2008), where some of these models are compared. We just note that, during solar maximum conditions the entire volume of the heliosphere is filled with low-speed solar wind, while during solar minimum conditions, a high-speed solar wind evolves over the poles, i.e. above/below $\pm 35^{\circ}$ the ecliptic, and persists over a period of about three years as observed by the Ulysses spacecraft (see McComas et al. 2003).

Generally, the HMF, $\boldsymbol{B}$, in spherical polar coordinates, can be described by its radial $B_{\mathrm{r}^{-}}$, longitudinal $B_{\varphi^{-}}$, and latitudinal $B_{\vartheta}$-components:

$$
\boldsymbol{B}=B_{\mathrm{r}} \boldsymbol{e}_{\mathrm{r}}+B_{\varphi} \boldsymbol{e}_{\varphi}+B_{\vartheta} \boldsymbol{e}_{\vartheta}
$$

with the spherical polar coordinate unit vectors, and $\varphi \in\{-\pi . \pi\}$ and $\vartheta \in\{-\pi / 2, \pi / 2\}$ are the longitude and latitude, respectively.

\subsection{Analytic HMF representations}

The most commonly assumed HMF, the so-called Parker field $\boldsymbol{B}_{\mathrm{P}}$ (Parker 1958), is a modified dipole field of the Sun, with a radial component decreasing like $r^{-2}$, and a latitude-dependent azimuthal component decreasing with $r^{-1}$ vanishing with $\cos \vartheta$ over the heliospheric poles (e.g. Parker 1958), see also for observational data Forsyth et al. (2002). To avoid the $r^{-2}$ behavior at the heliospheric poles, Smith \& Bieber (1991) added a small term decreasing with $r^{-1}$ to the $B_{\varphi}$-component, resulting in the modified Parker field $\boldsymbol{B}_{\mathrm{mP}}$.

Taking the differential rotation of the Sun's surface into account, Fisk (1996) modeled the motion of the HMF footpoints at the Sun. Due to that motion, the so-called Fisk field $\boldsymbol{B}_{\mathrm{F}}$ also has a $\vartheta$-component that is not present in the other two described HMFs. Because the Fisk field is restricted to a constant solar wind speed, Schwadron (2002); Schwadron et al. (2008) extended it to the case where the solar wind speed can be latitudedependent, i.e. for high-speed streams at high latitudes, and lowspeed flow in the ecliptic. The latter is called the modified Fisk field $\boldsymbol{B}_{\mathrm{mF}}$. The Fisk field is used in the hybrid representation by Burger \& Hitge (2004), where a Parker-like field dominates in the ecliptic and over the poles, while in between a Fisk-like field contributes most to the HMF.

The analytic representations of these fields are given by:

- the Parker field $\boldsymbol{B}_{\mathrm{P}}$ :

$$
B_{\mathrm{P}, \mathrm{r}}=A \frac{b_{0}}{r^{2}}, \quad B_{\mathrm{P}, \varphi}=-B_{\mathrm{P}, \mathrm{r}} k\left(r-R_{\odot}\right) \cos \vartheta
$$

- the modified Parker field $\boldsymbol{B}_{\mathrm{mP}}\left(\delta=5 \times 10^{-2}\right)$ :

$$
\boldsymbol{B}_{\mathrm{mP}}=\boldsymbol{B}_{\mathrm{P}}-B_{\mathrm{P}, \mathrm{r}} \delta r \cos \vartheta \boldsymbol{e}_{\varphi}
$$

- the hybrid Fisk field $\boldsymbol{B}_{\mathrm{F}}$ :

$$
\begin{aligned}
B_{\mathrm{F}, \mathrm{r}}= & B_{\mathrm{P}, \mathrm{r}}, \quad B_{\mathrm{F}, \vartheta}=B_{\mathrm{P}, \mathrm{r}} r \frac{F_{s}(\vartheta)}{v_{\mathrm{sw}}} \omega \sin \beta \\
B_{\mathrm{F}, \varphi}= & B_{\mathrm{P}, \varphi}+B_{\mathrm{P} . \mathrm{r}} \frac{r}{v_{\mathrm{sw}}}\left(\omega F_{s} \cos \beta \cos \vartheta\right. \\
& +\omega F_{s} \sin \beta \sin \vartheta \cos \left(\varphi+\varphi_{v}\right) \\
& \left.-\omega \frac{d F_{s}}{d \vartheta} \sin \beta \cos \vartheta \cos \left(\varphi+\varphi_{v}\right)\right)
\end{aligned}
$$

- and its modification by Schwadron (2002) $\boldsymbol{B}_{\mathrm{mF}}$ where the solar wind speed can be a function of latitude:

$$
\boldsymbol{B}_{\mathrm{mF}}=\boldsymbol{B}_{\mathrm{F}}+\frac{B_{\mathrm{F}, \vartheta}}{v_{\mathrm{sw}}(\vartheta)} \frac{\mathrm{d} v_{\mathrm{sw}}(\vartheta)}{\mathrm{d} \vartheta} \boldsymbol{e}_{\mathrm{r}}
$$

with

$k=\frac{\Omega_{\odot}}{v_{\mathrm{sw}}(\vartheta)}, \quad \varphi_{v}(\vartheta)=\frac{\Omega_{\odot} r}{v_{\mathrm{sw}}(\vartheta)}=k r$

with $b_{0}=B\left(r_{0}\right) r_{0}^{2}, B\left(r_{0}\right)$ the magnetic field strength in $\mu \mathrm{G}$ at $r_{0}=$ $60 R_{\odot} \approx 0.28 \mathrm{AU}\left(R_{\odot}\right.$ is the solar radius), $\Omega_{\odot}=2.9 \times 10^{-6} \mathrm{~Hz}$ is the averaged solar rotation frequency, and $v_{\mathrm{sw}}(\vartheta)$ the solar wind speed. The constant $A= \pm 1$ in Eq. (2) indicates the different field directions between the northern and southern hemispheres (Burger \& Hitge 2004). The transition function

$$
\begin{aligned}
F_{s}(\vartheta)= & f_{s}^{2}(\vartheta) \\
f_{s}(\vartheta)= & \tanh \left[\delta_{\mathrm{p}}\left(\frac{\pi}{2}-\vartheta\right)\right]-\tanh \left[\delta_{\mathrm{p}}\left(\vartheta+\frac{\pi}{2}\right)\right]+\tanh \left[\delta_{\mathrm{e}} \vartheta\right] \\
\frac{\mathrm{d} F_{s}(\vartheta)}{\mathrm{d} \vartheta}= & 2 f_{s}(\vartheta)\left(\frac{\delta_{\mathrm{e}}}{\cosh ^{2}\left[\delta_{\mathrm{e}} \vartheta\right]}-\frac{\delta_{\mathrm{p}}}{\cosh ^{2}\left[\delta_{\mathrm{p}}\left(\vartheta+\frac{\pi}{2}\right)\right]}\right. \\
& \left.-\frac{\delta_{\mathrm{p}}}{\cosh ^{2}\left[\delta_{\mathrm{p}}\left(\vartheta-\frac{\pi}{2}\right)\right]}\right)
\end{aligned}
$$

describes the relative contributions of the Fisk and Parker fields to the HMF; e.g., for $F_{s}=0$ it is a pure Parker field, while for $F_{s}=1$ it is a pure Fisk field as defined by Burger \& Hitge (2004), but see also Burger et al. (2008) for a different definition. The values for the angle between the rotational axis and the socalled virtual axis of the $\operatorname{Sun} \beta=30^{\circ}$ and the differential rotation rate $\omega=\frac{\Omega_{\odot}}{4}$ are taken from Burger \& Hitge (2004). The distance $r_{0}=60 R_{\odot}$ is in the region where the magnetic field is frozen into the solar wind. The parameters $\delta_{\mathrm{p}}=5$ and $\delta_{\mathrm{e}}=4$ determine the contribution of the Fisk- and Parker fields over the poles and in the ecliptic.

The function $F_{s}$ and its derivative - required for the divergence-free constraint of the magnetic field - is presented in Fig. 1, where it can be seen that the derivative is large close to the poles and the ecliptic, so, the $\varphi$-component of the hybrid Fisk fields only vanishes directly at the poles and in the ecliptic. For a Parker field with constant solar wind speed $v_{\mathrm{sw}}=400 \mathrm{~km} \mathrm{~s}^{-1}$, the parameter $k=\frac{\Omega_{\odot}}{v_{\mathrm{sw}}}=1.08025 \mathrm{AU}^{-1}$, while for a Fisk field a constant solar wind speed $v_{\mathrm{sw}}=600 \mathrm{~km} \mathrm{~s}^{-1}$ is commonly taken (Fisk 1996; Burger \& Hitge 2004; Burger 2005; Burger et al. 2008), and at $1 \mathrm{AU}$ the value $\varphi_{v}=\frac{2}{3} k$. 


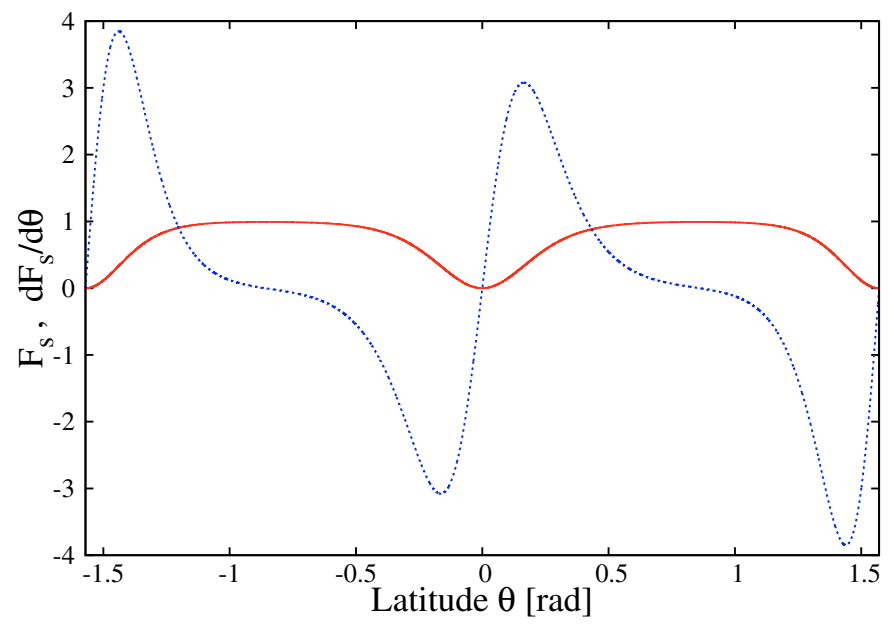

Fig. 1. The transition function $F_{s}(\vartheta)$ (red solid line) and its derivative (blue dotted line).

The low-speed solar wind typically has $v_{\mathrm{sw}}=400 \mathrm{~km} \mathrm{~s}^{-1}$, while the high-speed wind in polar regions (i.e. at latitudes above/below $\left.\vartheta_{0}= \pm 35^{\circ}\right)$ has twice that value $\left(800 \mathrm{~km} \mathrm{~s}^{-1}\right)$. Thus during solar minimum conditions the $k$ at high latitudes has to be reduced by a factor two. To avoid an unsteady sharp transition at $\vartheta_{0}= \pm 35^{\circ}$, we introduce the following function $f(\vartheta)$ :

$f(\vartheta)=\frac{1}{2}\left(\tanh \left(\Delta\left(\vartheta+\vartheta_{0}\right)\right)-\tanh \left(\Delta\left(\vartheta-\vartheta_{0}\right)\right)\right)$

where $\vartheta$ is given in radian and $\Delta=10$ is chosen, which corresponds to a width of the transition region from a slow to fast solar wind of $\approx 12^{\circ}$ and $v_{\mathrm{sw}}=400(2-f(\vartheta)) \mathrm{km} \mathrm{s}^{-1}$. fields.

In the next section we discuss the differences between these

\section{The angle with respect to the Parker field}

The Parker-like fields and the modified hybrid Fisk field are calculated using high/low-speed streams, while a constant solar wind speed $v_{\mathrm{sw}}=600 \mathrm{~km} \mathrm{~s}^{-1}$ is chosen for the hybrid Fisk field. To compare three-dimensional vectors we can either do it by components or use the angle relative to a known structure. Other options may exist, but we prefer the latter and will use the Parker field as the known structure, because it consists of Archimedian spirals with winding angles decreasing towards the heliospheric poles. In Figs. 2 and 3 we have plotted the angles $\boldsymbol{\xi}$ between the Parker field $\boldsymbol{B}_{\mathrm{P}}$ and the other three fields $\boldsymbol{B}_{\mathrm{mP}}, \boldsymbol{B}_{\mathrm{F}}$ and $\boldsymbol{B}_{\mathrm{mF}}$, i.e.,

$\xi_{i}=\arccos \left(\frac{\boldsymbol{B}_{\mathrm{P}} \cdot \boldsymbol{B}_{i}}{B_{\mathrm{P}} B_{i}}\right)$

(with $i \in\{m P, F, m F\}$ ) at different heliospheric distances and in a projection of the heliospheric coordinates $(\varphi, \vartheta)$. Because $B_{0} r_{0}^{2}$ is a common factor, it does not influence the results. While we present $\xi_{i}$ in a Hammer projection, a Lambert projection of the polar regions is shown in Fig. 3 to resolve the heliocentric poles. In Figs. 2 and 3, heliospheric distances greater than $\approx 100$ AU may be academic for all directions. Because the termination shock distance is latitude- and longitude-dependent, the results in these figures are only valid for directions where the shown heliocentric distances are less than the termination shock distance.

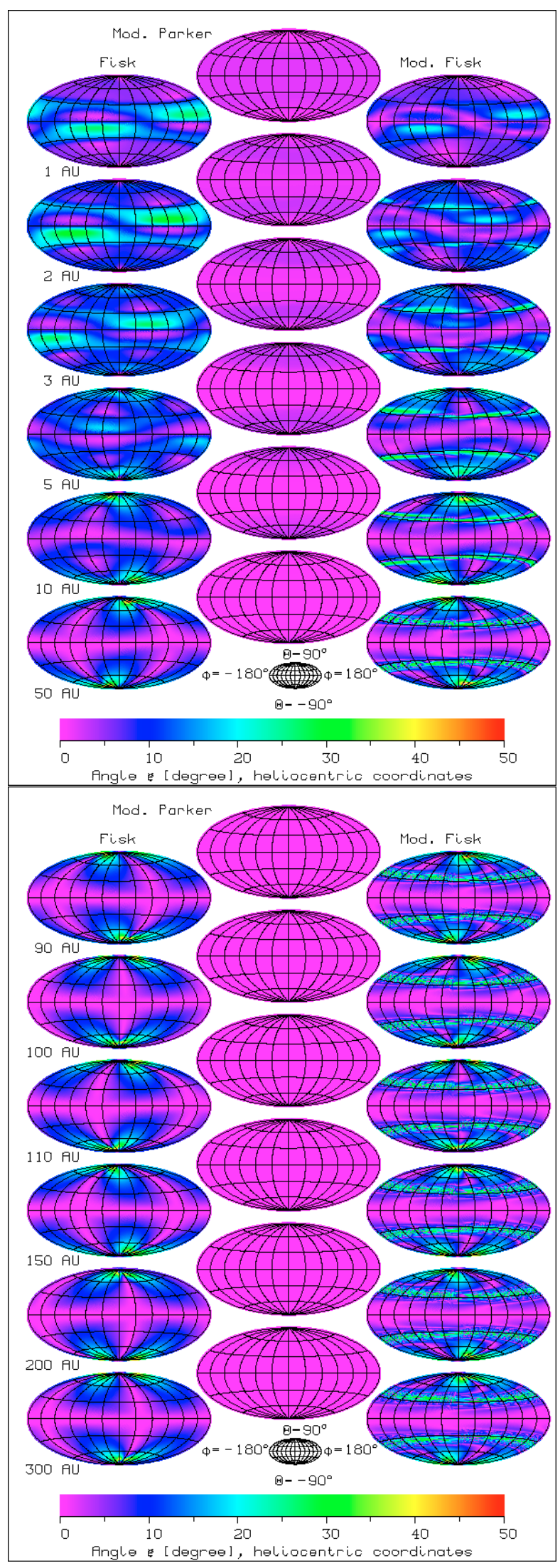

Fig. 2. The Hammer projection (view from the Sun outwards) of the angle $\xi$ in heliocentric coordinates. On the left the hybrid Fisk field is represented, in the middle the modified Parker field, and on the right the modified hybrid Fisk field. Left panel: from top to bottom the heliocentric distances varies from: $r=1,2,3,5,10$, to $50 \mathrm{AU}$. Right panel: $r=90,100,110,150,200$, and $300 \mathrm{AU}$. 


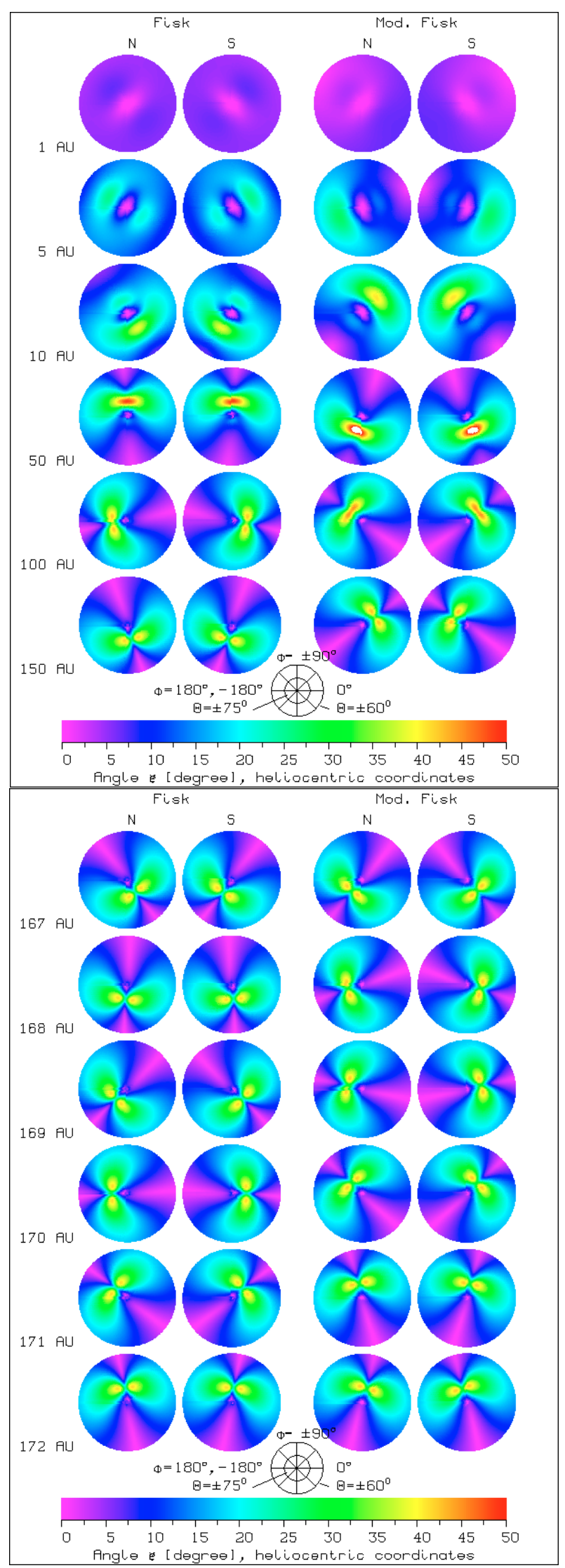

Fig. 3. Left panel: the north (left) and south (right) pole of the angle $\xi$ for the Fisk (left) and the modified Fisk field (right) in a Lambert projection (view from outside towards the Sun). Right panel: angle $\xi$ in the vicinity of the termination shock above the poles.
With the procedure described above we lose the information on field vector orientations. To maintain the three-dimensional information, the scalar product can be calculated with respect to the unit vectors, for instance in spherical coordinates $\boldsymbol{e}_{\mathrm{r}}, \boldsymbol{e}_{\varphi}, \boldsymbol{e}_{\vartheta}$, which will be helpful when studying the three-dimensional propagation of cosmic rays, e.g. the elements of the corresponding diffusion tensor.

In both panels of Fig. 2, the modified Parker field is shown in the middle column. For small heliocentric distances, the SmithBieber term in the $\varphi$-component is large enough to contribute to the scalar product in Eq. (7), while at greater distances, it vanishes, and both magnetic field vectors $\boldsymbol{B}_{\mathrm{P}}$ and $\boldsymbol{B}_{\mathrm{mP}}$ mainly have a $\varphi$-component, hence are parallel. Therefore, the small nonzero angles faintly visible in the top four panels of the middle columns of Figs. 2 vanish with increasing distances. Because the modified Parker field $\boldsymbol{B}_{\mathrm{mP}}$ behaves almost like the Parker field $\boldsymbol{B}_{\mathrm{P}}$, it is not further discussed in the following.

The hybrid Fisk field $\boldsymbol{B}_{\mathrm{F}}$ is shown in the left columns of both panels of Fig. 2, while the modified hybrid Fisk field $\boldsymbol{B}_{\mathrm{mF}}$ is presented in the right columns. In Fig. 3 the north pole (i.e. $\vartheta=$ $\left.90^{\circ}\right)$ and south pole $\left(\vartheta=-90^{\circ}\right)$ are displayed for the hybrid Fisk $\boldsymbol{B}_{\mathrm{F}}$ and the modified hybrid Fisk $\boldsymbol{B}_{\mathrm{mF}}$ field, respectively.

At $1 \mathrm{AU}$, the angles $\xi_{\mathrm{F}, \mathrm{mF}}$ between the Fisk-like fields $\boldsymbol{B}_{\mathrm{F}}, \boldsymbol{B}_{\mathrm{mF}}$ at low latitudes and the Parker field $\boldsymbol{B}_{\mathrm{P}}$ are small except in some confined regions close to the equator. These differences are due to the longitudinal structure of the former which is amplified by the derivative term of the transition function near the equatorial plane, see also Fig. 1. The areas at the poles with large angles $\xi_{\mathrm{F}, \mathrm{mF}}$ (see also Fig. 3) increase with increasing heliocentric distance $r$ up to an asymptotic size of about $50^{\circ}$. The highest values of $\xi_{\mathrm{F}, \mathrm{mF}}$ occur at distances of approximately 50 AU. The angle is slightly greater for the modified Fisk field. Due to the similarity of the Fisk and the Parker fields at small heliocentric distances and high latitudes (see also Sternal et al. $2008 \mathrm{~b}) \xi_{\mathrm{F}, \mathrm{mF}}<20^{\circ}-30^{\circ}$ for distances $r<5 \mathrm{AU}$. At heliocentric latitudes below $60^{\circ}$ the angles $\xi_{\mathrm{F}, \mathrm{mF}}$ are generally limited to about $30^{\circ}$ for all distances. The modified Fisk-field $\boldsymbol{B}_{\mathrm{mF}}$ shows bands around the transition regions at $\vartheta= \pm 35^{\circ}$ in which the angle $\xi_{\mathrm{mF}}$ shows a "chaotic" behavior, i.e. varies strongly on small spatial scales.

With increasing distances, the Fisk-like fields show stripes from pole to pole in which they are almost parallel to the Parker field, i.e. $\xi_{\mathrm{F}} \approx \xi_{\mathrm{mF}} \approx 0^{\circ}$. As a consequence of the radial variation of the longitudinal structure of the Fisk-like fields, these stripes "rotate" anti-clockwise with increasing heliocentric distance $r$; i.e. with increasing distance the parallelism is shifted to different longitudes. In the right panel of Fig. 3 it can be seen that such a " $\xi$-rotation" exceeds $\approx 30^{\circ}$ per AU for the hybrid Fisk field $\boldsymbol{B}_{\mathbf{F}}$ and is a little less for the modified hybrid Fisk field $\boldsymbol{B}_{\mathrm{mF}}$. In this figure the polar regions of the fields $\boldsymbol{B}_{\mathrm{F}, \mathrm{mF}}$ are shown, the south and north poles of $\boldsymbol{B}_{\mathrm{F}}$ in the left two columns of each panel, and those of $\boldsymbol{B}_{\mathrm{mF}}$ in the two right columns. These fields exhibit a north-south asymmetry at a given distance as a consequence of the tilt of the magnetic axis relative to the rotational axis. To illustrate the radial variation of the angle $\xi_{i}$, a distance range around the polar termination shock is shown in $1 \mathrm{AU}$ steps in the right panel of Fig. 3. One can easily see the strong radial dependence of $\xi_{i}$ implying an equally strong dependence of the injection efficiency on the location and dynamics of the termination shock. As a further illustration, the large scale variation in $\xi_{\mathrm{F}}$ is visualized with a meridional cut displayed in Fig. 4.

From the above discussion, one should expect a north-south as well as an east-west asymmetry for Fisk-like field configurations at the different positions of the termination shock, as 
K. Scherer et al.: Comparison of analytic heliospheric magnetic fields

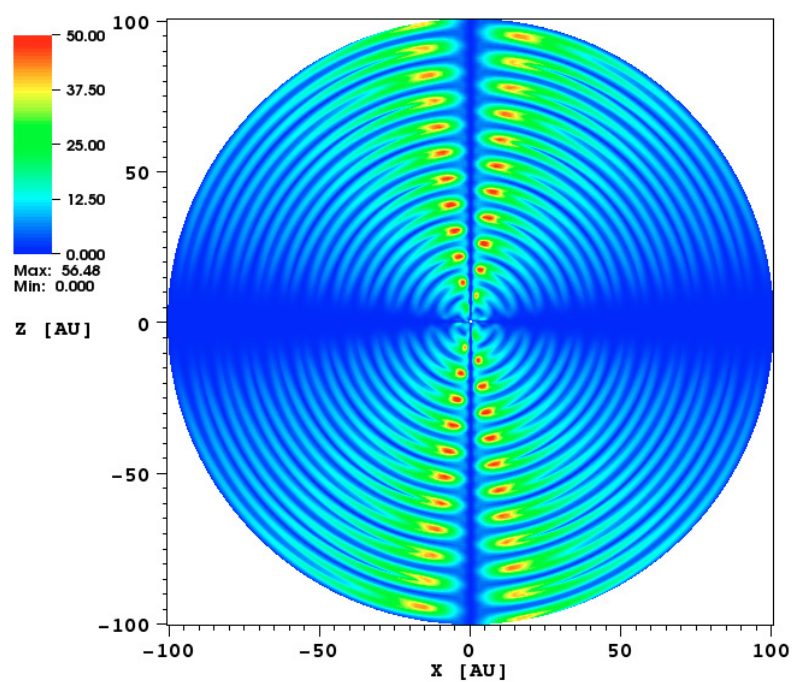

Fig. 4. The angle between the hybrid Fisk- and Parker-field in a meridional plane, the large spatial variation in the regions where the Fisk-type field is dominant.

observed by Voyager 1 and 2 spacecraft, i.e. at 94 and 84 AU, respectively (Stone et al. 2005, 2008). Thus, the recent Voyager observations (Richardson et al. 2008), which suggest that the termination shock may be North-South-asymmetric, should not be a surprise.

The small asymmetry is interpreted by the cited authors as a deviation of the interstellar magnetic field vector from the inflow direction. However, the latter mainly affects the shape of the heliopause (Opher et al. 2009; McComas et al. 2009a; Schwadron et al. 2009) and that of the bow shock, but that of the termination shock only weakly (Pogorelov et al. 2008). In view of the above discussion, the observed asymmetry may partly be explained by that of the Fisk-type fields, which need to be incorporated into MHD models of the heliosphere-interstellar medium interaction. In any case, the termination shock is not only "geometrically" asymmetric - as observed by the Voyagers, see, e.g., Stone et al. (2008) - but it also shows a "magnetic" asymmetry, i.e. a northsouth difference in the angle between the Fisk-like fields and the shock normal as is quantified in the following.

\section{Representation of the heliospheric termination shock}

For the shock surface we use the 3D-model of the solar wind and interstellar medium interaction during solar minimum by Borrmann \& Fichtner (2005) including high speed streams over the polar regions. The intersections of the shock surface with the equatorial $x-y$-plane and the meridional $x-z$-plane are shown in Fig. 5.

\subsection{Analytic triaxial ellipsoid}

To study the effects of the different fields $\boldsymbol{B}_{i}$ qualitatively at the termination shock, we first use an analytical representation of the shock surface. Therefore, we follow the description by Scherer \& Fahr (2009) and only briefly repeat the main idea that the three-dimensional termination shock surface is described as a triaxial ellipsoid (Fig. 5). The parameters of the ellipsoid are $a=$ $195, b=170, c=194.5 \mathrm{AU}$, so that the eccentricities are given by $\epsilon_{1}=\sqrt{1-b^{2} / a^{2}}=0.49, \epsilon_{2}=\sqrt{1-c^{2} / a^{2}}=0.07$, and the semi-axes $a, b, c$ are oriented along the $x, y, z$-axes in a Cartesian
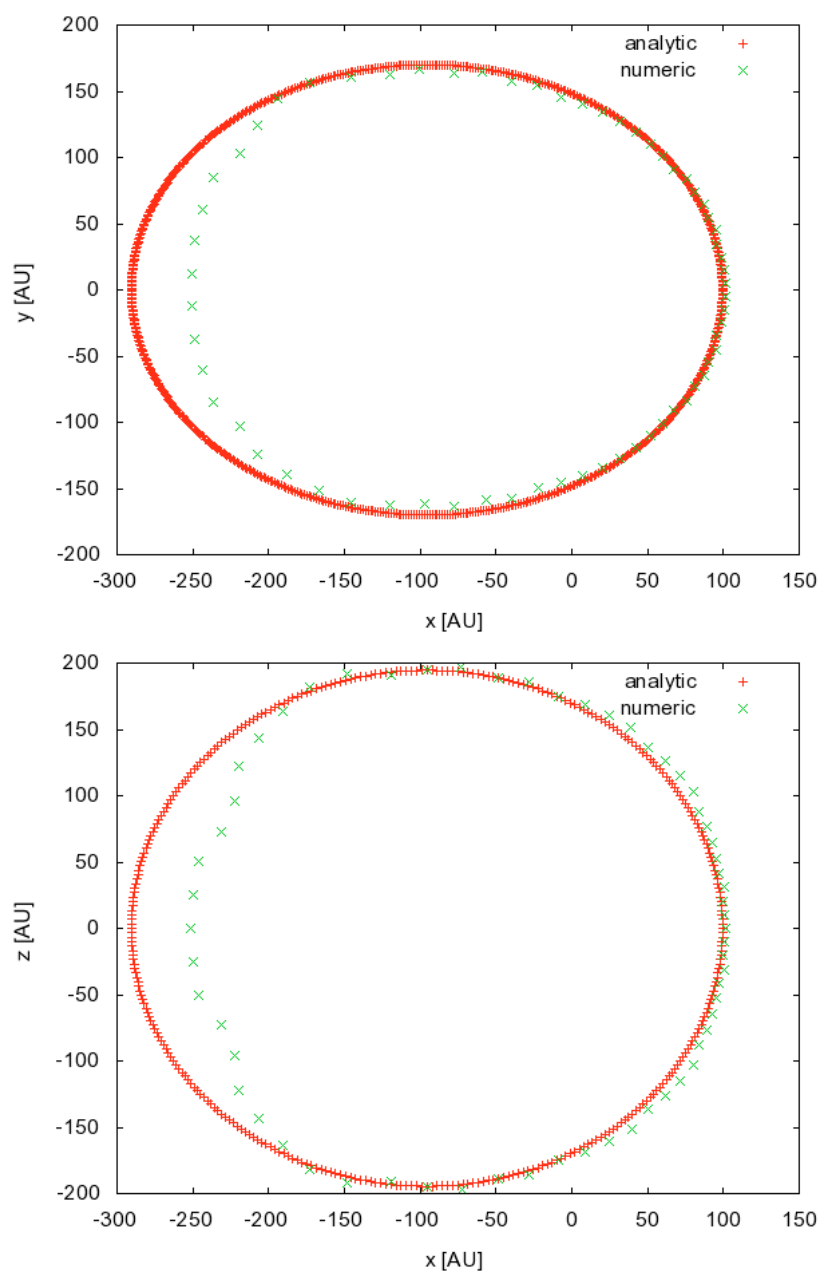

Fig. 5. The fit (red crosses) to the model results (green crosses) by Borrmann \& Fichtner (2005). The equatorial plane is shown in the left panel and in the right the meridional one.

coordinate system, respectively. The interstellar medium flows from the positive to the negative $x$-axis, with the ecliptic in the $x-y$-plane, and the polar regions are directed along the $z$-axis. In this analytic approach it is straightforward to calculate the normal vector $\boldsymbol{n}_{a}$ to the shock surface needed for the computation of the magnetic tilt angle.

\subsection{Numerical model}

The deviations of the fitted ellipsoid from the actual shock surface are relatively large in the tail regions, see Fig. 5. Therefore, we calculated the normal vector to the numerically determined shock surface in the following way: we computed the local orientation of the surface using a midpoint and pairs of its neighboring points. From eight neighbors of a given surface point, we obtained eight representations of the normal vector. After averaging these representations, we derived a good approximation of the normal vector $\boldsymbol{n}_{n}$ at this location.

In the following we compare the magnetic tilt angles for the large-scale magnetic field structures using both the analytically and numerically obtained normal vectors.

\section{The magnetic tilt angle}

We determine the tilt angles $\psi$ as the scalar product between the shock normal $\boldsymbol{n}$ and the Fisk-like magnetic fields $\boldsymbol{B}_{\mathrm{F}, \mathrm{mF}}$ at 

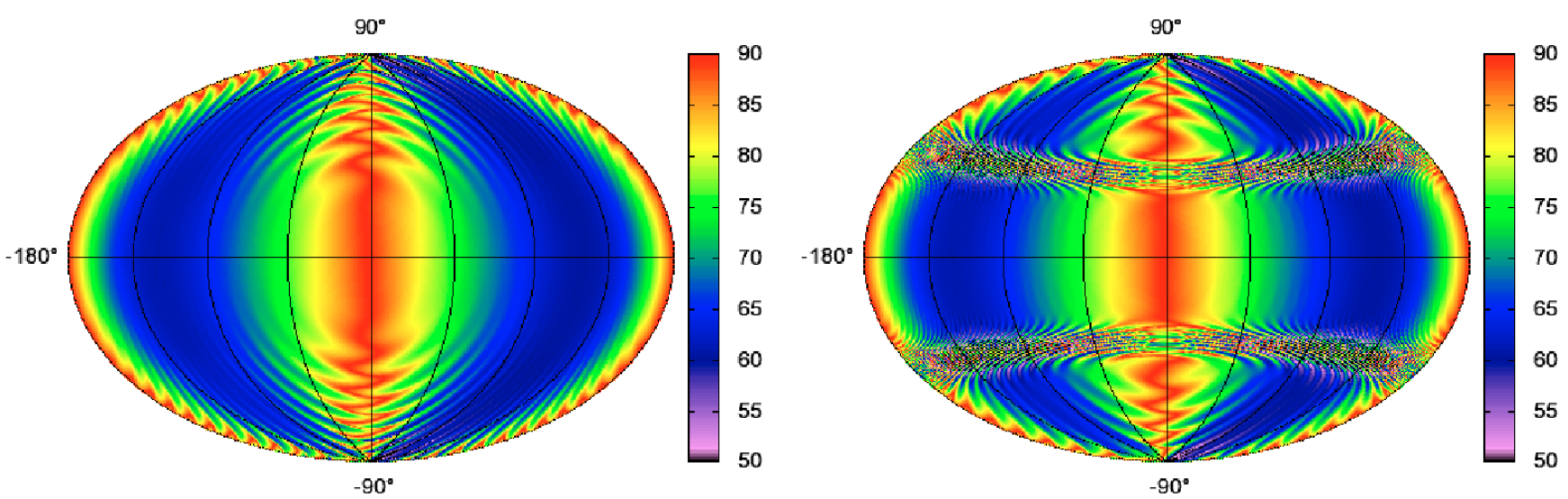

Fig. 6. The magnetic tilt angle of the Fisk (left) and the modified Fisk field (right) at the analytically approximated termination shock.
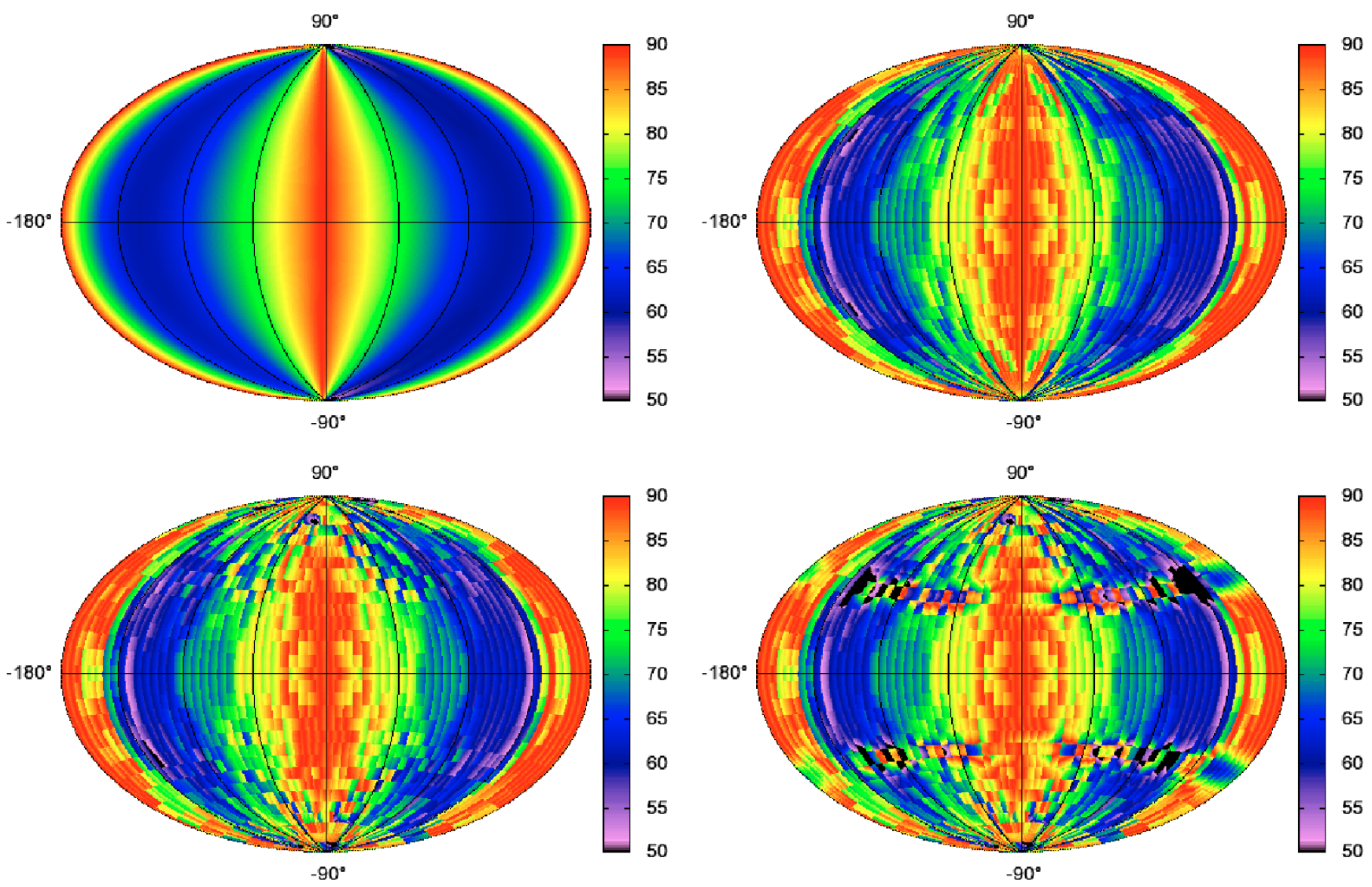

Fig. 7. The tilt angle for the Parker field in the analytic representation is shown in the upper left panel. For the numerical case the tilt angle of the Parker field is shown in the upper right, for the Fisk field in the lower left, and for the modified Fisk field in the lower right.

the shock surface, while those for the Parker field are discussed in detail in Scherer \& Fahr (2009). The former are presented in Fig. 6 for the analytically and in Fig. 7 for numerically calculated normal vectors, respectively. In Fig. 6 it can be seen that a strip from the poles through the nose direction around $\left(\varphi=0^{\circ}\right)$, like in the Parker field, exists for the Fisk-like fields where it takes values about $\psi=90^{\circ}$.

As was shown by Scherer \& Fahr (2009) the magnetic tilt angle $\psi$ can be as low as $60^{\circ}$ in the heliospheric flanks for the Parker field $\boldsymbol{B}_{\mathrm{P}}$, and exhibits a modest "east-west" asymmetry as a result of the different orientation of the field and the shock normal. A similar behavior can be observed in Fig. 6 for the Fisk-like fields. The tilt angles for the fields $\boldsymbol{B}_{\mathrm{F}, \mathrm{mF}}$ show a "background" variation related to the varying distances $r$ to the ellipsoid surface. The "filamentary" structures obtained for the analytical representation of the shock surface are a consequence of the complicated field geometry, however, it is only weakly visible in the numerical case, because the resolution of the underlying numerical model is limited. In that case, the differences between the hybrid Fisk field $\boldsymbol{B}_{\mathrm{F}}$ and the Parker field $\boldsymbol{B}_{\mathrm{P}}$ are small (see upper left panel of Fig. 7) and are only visible in the analytical case as a fine structure. The modified hybrid Fisk field $\boldsymbol{B}_{\mathrm{mF}}$ 
shows an additional band of highly varying tilt angles in the transition region from the slow to the fast solar wind in bands around $\vartheta= \pm 35^{\circ}$.

The tilt angle of the Fisk-like fields is quite similar to that of the Parker field, so, the injection efficiency of the PUIs into the ACR acceleration process is quite similar to what is described in Scherer \& Fahr (2009). The exception is the modified hybrid Fisk field, which shows the band-like structure, in which the magnetic tilt angle changes strongly on small spatial scales, allowing for alternating higher or lower injection efficiencies in this structure (see Fig. 7, right panel).

Because the Parker field $\boldsymbol{B}_{\mathrm{P}}$ is the prevailing heliospheric magnetic field for solar maximum conditions, solar activity effects are not considered here. Scherer \& Fahr (2009) already showed, however, that the injection conditions are fairly good across the entire termination shock surface during solar maximum.

\subsection{Implications for cosmic ray propagation}

The influence of Fisk-like fields $\boldsymbol{B}_{\mathrm{F}, \mathrm{mF}}$ on the propagation of energetic particles in the heliosphere, and especially on the acceleration processes in the heliosheath, is not easily accessible, because these fields change with heliocentric distance, longitude, and latitude and, therefore, the corresponding diffusion tensor will change analogously. In order to quantitatively treat the cosmic ray transport for which gradient and curvature drifts, as well as spatial diffusion are significant processes, it is necessary to calculate a supposedly fully anisotropic diffusion tensor and to re-derive the drift terms for the different field configurations. This would go far beyond the scope of this paper, so the reader is referred to Burger et al. (2008) and Sternal et al. (2008b). Instead, we briefly address possible effects that an enhanced PUI production may have on the global distribution of Energetic Neutral Atoms (ENAs).

\subsection{Implications for ENA fluxes}

The injection efficiency describes the fraction of PUIs at the termination shock, which can be accelerated to ACRs. As discussed above, the injection efficiency of PUIs into the Fermi-I process producing the anomalous component is expected to be structured only weakly depending on the magnetic field configuration (see Fig. 7).

If the injection efficiency in a given direction is sufficiently high, there might be lower ENA fluxes at PUI energies from there because of the "extraction" of PUIs to the ACR component. The effect of the above-mentioned fine structure, however, might be too small to be detected with the IBEX mission (McComas et al. 2009b).

\section{Conclusions}

We calculated the angles $\xi_{\mathrm{mP}, \mathrm{F}, \mathrm{mF}}$ between the modified Parker field $\boldsymbol{B}_{\mathrm{mP}}$, the Fisk field $\boldsymbol{B}_{\mathrm{F}}$, the modified Fisk field $\boldsymbol{B}_{\mathrm{mF}}$ and the Parker field $\boldsymbol{B}_{\mathrm{P}}$ and discussed their variations and deviations from each other with heliocentric distance, latitude, and longitude. The largest $\xi \geq 50^{\circ}$ occur at high latitudes. Due to the longitudinal structure of the Fisk-like fields there are, however, also stripes at certain longitudes reaching from pole to pole, where the fields are parallel to the Parker field (i.e. $\xi=0^{\circ}$ ). These stripes rotate anti-clockwise with increasing heliocentric distance.
We continued to expand our previous large scale threedimensional semi-analytic approach to study the tilt angle between the vectors of different magnetic fields and the shock surface normal. The main result is that the differences in the injection efficiency for the Parker field and for its modification, as well as for the hybrid Fisk field are negligible. We want to point out that the hybrid Fisk field is only consistent with a constant solar wind speed, while all other fields studied here (Parker-like and modified hybrid Fisk fields) can take the fast solar wind streams into account at high latitudes during solar minimum conditions.

For the $\boldsymbol{B}_{\mathrm{P}, \mathrm{mP}, \mathrm{F}}$ fields we conclude that, during solar minimum conditions, the favorable acceleration regions are always the flanks of the 3D-heliosphere. This finding is consistent with the measurements by both Voyager spacecraft, which did not find any evidence of ACR acceleration closer to the upwind direction.

We summarize the main conclusions drawn from these above considerations of these magnetic fields in the following:

1. For Fisk-like fields $\boldsymbol{B}_{\mathrm{F}, \mathrm{mF}}$ there exists an east-west and northsouth asymmetry.

2. The deviations of the two Fisk-like fields $\boldsymbol{B}_{\mathrm{F}, \mathrm{mF}}$ directions from that of the Parker field $\boldsymbol{B}_{\mathrm{P}}$ can be significant at all heliocentric positions and large in polar regions.

3. For all fields discussed here, the efficiency of injection of PUIs into the Fermi-I acceleration process at the termination shock is expected to be essentially the same, because the deviations in the different tilt angles at the termination shock are only visible in a fine structure, which is most probably not accessible to observations. The only distinct features are relatively narrow bands of strong tilt angle variations occurring for the modified Fisk field in the transition region from a slow to a fast wind.

4. During solar minimum, the favorable acceleration regimes for ACRs are in the flanks of the heliosphere.

Acknowledgements. H.F. and K.S. are grateful to the Deutsche Forschungsgemeinschaft (DFG) for funding the Heliocauses project and to the Bundesministerium für Bildung und Forschung (BMBF) for supporting the bilateral cooperation with South Africa. R.A.B. acknowledge the partial financial support of the SA National Research Foundation.

\section{References}

Burger, R. A. 2005, Adv. Space Res., 35, 636

Burger, R. A., \& Hitge, M. 2004, ApJ, 617, L73

Borrmann, T., \& Fichtner, H. 2005, Adv. Space Res., 35, 2091

Burger, R. A., Krüger, T. P. J., Hitge, M., \& Engelbrecht, N. E. 2008, ApJ, 674, 511

Burlaga, L. F., Ness, N. F., Wang, Y., \& Sheeley, N. R. 2002, J. Geophys. Res., 107,1410

Ebert, R. W., McComas, D. J., Elliott, H. A., Forsyth, R. J., \& Gosling, J. T. 2009, J. Geophys. Res., 114, 1109

Fahr, H. J., Kausch, T., \& Scherer, H. 2000, A\&A, 357, 268

Fahr, H. J., Scherer, K., Potgieter, M. S., \& Ferreira, S. E. S. 2008, A\&A, 486, L1

Ferreira, S. E. S., Potgieter, M. S., \& Scherer, K. 2007, ApJ, 659, 1777

Fisk, L. A. 1996, J. Geophys. Res., 101, 15547

Forsyth, R. J., Balogh, A., \& Smith, E. J. 2002, J. Geophys. Res., 107, 1405

Giacalone, J., \& Jokipii, J. R. 2004, ApJ, 616, 573

Giacalone, J., Jokipii, J. R., \& Matthaeus, W. H. 2006, ApJ, 641, L61

Heerikhuisen, J., Pogorelov, N. V., Florinski, V., Zank, G. P., \& Le Roux, J. A. 2008, ApJ, 682, 679

Hitge, M., \& Burger, R. A. 2010, Adv. Space Res., 45, 18

Izmodenov, V. V., Malama, Y. G., \& Ruderman, M. S. 2008, Adv. Space Res., 41,318 
Jokipii, J. R., \& Kota, J. 1989, Geophys. Res. Lett., 16, 1

McComas, D. J., Elliott, H. A., Schwadron, N. A., et al. 2003, Geophys. Res. Lett., 30, 24

McComas, D. J., Allegrini, F., Bochsler, P., et al. 2009a, Science, 326, 959

McComas, D. J., Allegrini, F., Bochsler, P., et al. 2009b, Space Sci. Rev., 146, 11

Mordvinov, A. V. 2008, Cosmic Res., 46, 314

Müller, H.-R., Florinski, V., Heerikhuisen, J., et al. 2008, A\&A, 491, 43

Murphy, N., Smith, E. J., \& Schwadron, N. A. 2002, Geophys. Res. Lett., 29, 220000

Mursula, K. 2007, Adv. Space Res., 40, 1034

Opher, M., Stone, E. C., \& Liewer, P. C. 2006, ApJ, 640, L71

Opher, M., Richardson, J. D., Toth, G., \& Gombosi, T. I. 2009, Space Sci. Rev., 143,43

Parker, E. N. 1958, ApJ, 128, 664

Pogorelov, N. V., Heerikhuisen, J., \& Zank, G. P. 2008, ApJ, 675, L41

Pogorelov, N. V., Borovikov, S. N., Zank, G. P., \& Ogino, T. 2009, ApJ, 696, 1478
Ratkiewicz, R. 2006, ASTRA, 2, 11

Richardson, J. D., Kasper, J. C., Wang, C., Belcher, J. W., \& Lazarus, A. J. 2008, Nature, 454, 63

Roberts, D. A., Giacalone, J., Jokipii, J. R., Goldstein, M. L., \& Zepp, T. D. 2007, J. Geophys. Res., 112, 8103

Scherer, K., \& Fahr, H.-J. 2003, A\&A, 404, L47

Scherer, K., \& Ferreira, S. E. S. 2005, ASTRA, 1, 17

Scherer, K., \& Fahr, H. 2009, A\&A, 495, 631

Schwadron, N. A. 2002, Geophys. Res. Lett., 29, 140000

Schwadron, N. A., \& McComas, D. J. 2003, Geophys. Res. Lett., 30, 110000

Schwadron, N. A., \& McComas, D. J. 2005, Geophys. Res. Lett., 32, 3112

Schwadron, N. A., Owens, M., \& Crooker, N. U. 2008, ASTRA, 4, 19

Schwadron, N. A., Bzowski, M., Crew, G. B., et al. 2009, Science, 326, 966

Smith, C. W., \& Bieber, J. W. 1991, ApJ, 370, 435

Sternal, O., Fichtner, H., \& Scherer, K. 2008a, A\&A, 477, 365

Sternal, O., Burger, A., Heber, B., et al. 2008b, Proc. Int. Cosmic Ray Conf., 451

Stone, E. C., Cummings, A. C., McDonald, F. B., et al. 2005, Science, 309, 2017

Stone, E. C., Cummings, A. C., McDonald, F. B., et al. 2008, Nature, 454, 71 